\title{
Desarrollo de piómetra y su relación con ovarios poliquísticos en hembras caninas
}

\author{
Luz Z. Duarte*, MVZ, Favio Sánchez, $M V Z_{1}$, Carolina Ortega, $M V Z_{1}$ \\ ${ }^{1}$ Grupo de Investigación en Pequeños Animales y Silvestres (GRIPAS), Facultad de Medicina Veterinaria y Zootecnia \\ Universidad Cooperativa de Colombia, Bucaramanga, Colombia
}

Recibido: 15 de enero del 2014. Aprobado: 30 de mayo del 2014.

*Autor de correspondencia: Luz Z. Duarte. Universidad Cooperativa de Colombia, Bucaramanga, Colombia, calle 30A \# 33-51. Teléfono: (+57) 7 6854500, ext. 7072. Correo electrónico: luz.duarte@campusucc.edu.co

Cómo citar este artículo: Duarte LZ, Sánchez S, Ortega C. Desarrollo de piómetra y su relación con ovarios poliquísticos en hembras caninas. Spei Domus. 2014;10(20):17-22. doi: http://dx.doi.org/10.16925/sp.v10i20.886

Resumen. Introducción: el presente trabajo tuvo como objetivo encontrar una relación entre el desarrollo del complejo hiperplasia endometrial quística (HEQ)-piómetra y los ovarios poliquísticos, por medio de la observación y las mediciones macroscópicas de úteros de pacientes caninas diagnosticadas con piómetra, a las que se les hizo cirugía de ovariouterotomía para la resolución del problema. Métodos: dichos órganos fueron obtenidos en dos clínicas de Bucaramanga. Todas las variables registradas fueron tabuladas en Excel 2003 y analizadas mediante el programa Epi Info 7, a fin de hacer un análisis estadístico descriptivo univariable, una tabla de contingencia y una prueba de Fisher. Se correlacionó la presentación de piómetra y de HEQ con el hallazgo de ovarios poliquísticos (OP). Estadísticamente, no se encontró asociación entre estas variables, $(p>0,05)$. Resultados: la existencia de HEQ fue del 87,10\% del total de los casos con respecto a la frecuencia de ovarios poliquísticos, dado que sólo se encontró un valor correspondiente al 32,26\% sin relación estadística entre estas dos patologías. La literatura reporta que la etiología del complejo HEQ es por aumento de hormonas estrogénicas en la etapa del diestro [1] y este desequilibrio hormonal, a su vez, es responsable del desarrollo de ovarios poliquísticos. Conclusiones: por lo tanto, sería pertinente elaborar un estudio con mediciones hormonales de las pacientes caninas que sean diagnosticadas con piómetra y verificar los hallazgos macroscópicos de los casos.

Palabras clave: diestro, endometritis, estrógenos, exudados, progesterona, quistes.

\section{Pyometra and Polycystic Ovaries in Female Dogs}

Abstract. Introduction: the objective of this paper was to find a connection between cystic endometrial hyperplasia (CEH)-pyometra and polycystic ovaries through observation and macroscopic measurements of canine uterus in subjects with pyometra that underwent hysterectomy to solve the problem. Methods: such organs were obtained from two clinics in Bucaramanga. All the found variables were registered in Excel 2003 and analyzed with Epi Info 7, so as to develop an invariable descriptive statistics analysis, a contingency table and a Fisher's exact test. The pyometra and CEH were correlated with polycystic ovaries (PO). Statistically, there was no relation between such variables, $(p>0,05)$. Results: existence of CEH was $87.10 \%$ of the total cases in respect of the polycystic ovaries frequency, since only $32.26 \%$ was not found to be statistically related. Literature reports that the etiology of the CEH complex is caused by an increase in estrogenic hormones in the diestrus phase [1] and such hormone imbalance is in turn responsible for the development of polycystic ovaries. Conclusions: it would be thus appropriate to conduct studies with the hormone measurements in canine subjects with pyometra and verify the macroscopic findings of such cases.

Keyword: diestrus, endometritis, estrogens, exudated, progesterone, cysts.

\section{Desenvolvimento de piometra e sua relação com ovários policísticos em fêmeas caninas}

Resumo. Introdução: este trabalho teve o intuito de achar uma relação entre o desenvolvimento do complexo hiperplasia endometrial cística (HEQ)-piometra e os ovários policísticos, através da observação e as medições macroscópicas de úteros de pacientes caninas diagnosticadas com piometra, nas quais se fez cirurgia de ováriohisterectomia para a resolução do problema. Métodos: tais órgãos foram obtidos em duas clínicas de Bucaramanga. Todas as variáveis cadastradas foram tabuladas em Excel 2003 e analisadas com o programa Epi Info 7, para fazer a análise estatística descritiva univariável, uma tabela de contingência e um teste Fisher. Foram correlacionadas a apresentação de piometra e de HEQ com o achado de ovários policísticos (OP). Estatisticamente, não foi achada associação entre estas variáveis, $(p>0,05)$. Resultados: a existência de HEQ foi de $87,10 \%$ do total dos casos em relação à frequência de ovários policísticos, porque somente foi achado um valor correspondente a $32,26 \%$ sem relação estatística essas duas patologias. A literatura indica que a etiologia do complexo HEQ é por aumento de hormonas estrogênicas na fase do diestro [1] e esse desequilíbrio hormonal, por sua vez, é responsável do desenvolvimento de ovários policísticos. Conclução: portanto, seria apropriado fazer um estudo com mensurações hormonais das pacientes caninas que sejam diagnosticadas com piometra e constatar os achados macroscópicos dos casos.

Palavras-chave: diestro, endometrite, estrogênio, exsudados, progesterona, cistos. 


\section{Introducción}

El complejo hiperplasia endometrial quística (HEQ) corresponde a una serie de cambios que anteceden a la piómetra [2]. Morfológicamente, se presentan alteraciones en el endometrio que facilitan la infiltración de células inflamatorias, con engrosamiento de las paredes endometriales y dilatación de los cuernos uterinos por la colección del exudado. En el desarrollo de la HEQ, la presencia de un cuerpo lúteo en la estructura ovárica demuestra que la progesterona se encuentra involucrada en la etiopatogenia. Sin embargo, la relación entre los quistes ováricos y la HEQ es poco registrada; algunos autores reportan enfermedades asociadas a los quistes ováricos foliculares como la hiperplasia endometrial quística, la endometritis, la piómetra y los fibroleiomiomas del tracto genital [3].

A nivel nacional, no se reporta la prevalencia de la hiperplasia endometrial y los ovarios poliquísticos, quizá solo algunos casos aislados, como lo expusieron Albarracín et al. [4]. Estos autores reportan un caso de OP y HEQ grado III, en el que se menciona la hiperplasia y el desarrollo de piómetra sin establecer la relación de los ovarios poliquísticos en el complejo endometrial.

Por su parte, Reynoso [5] desarrolló un estudio en el que analizó macroscópicamente los úteros obtenidos con posterioridad al procedimiento quirúrgico de ovariohisterectomía de pacientes con piómetra o tumores mamarios, y evidenció quistes ováricos en el $68 \%$, tumores ováricos en el $43 \%$ y HEQ en el $75 \%$ de los casos, sin hacer una asociación estadística. Este estudio se llevó a cabo con el fin de establecer la casuística del desarrollo de la piómetra y del complejo hiperplasia endometrial quística y su relación con ovarios poliquísticos [4], en un período de seis meses.

La piómetra es causa común de enfermedad en hembras caninas enteras, pues se reporta hasta en $6 \%$ [6] de todos los casos atendidos en clínicas veterinarias. Se observa a menudo que se realizan ovariohisterectomías de urgencia por llegada de pacientes con piómetra sin antecedentes o sin una etiología clara. La recopilación de los signos y la presencia del útero inflamado y lleno de líquido por ecografía o radiografía abdominal confirman el diagnóstico [7]. No se elaboran reportes de la cantidad de casos para identificar la relación de los ovarios poliquísticos con el desarrollo de piómetra.

Los quistes ováricos son estructuras ováricas de tipo folicular que surgen debido al fracaso de la ovulación; normalmente, miden más de $2 \mathrm{~mm}$ de diámetro y persisten en ausencia del cuerpo lúteo. Durante diez días o más, los quistes foliculares tienen paredes finas y son estructuras únicas, múltiples o multilobuladas presentes en uno o ambos ovarios. Los quistes parcialmente luteinizados tienden a ser estructuras unilaterales simples con paredes más gruesas, debido a la presencia de tejido lúteo [8].

Los reportes de ovarios poliquísticos son escasos y la literatura indica que se desconocen las causas exactas de la mayoría de quistes que se presentan. En los casos en que los quistes son foliculares funcionales, pueden producir estrógenos y progesterona, lo que podría generar en el caso de la condición de la HEQ-piómetra, una disminución de la actividad miometrial, facilitando la retención de líquido y favorecer la hiperplasia epitelial [7].

\section{Fisiopatología del complejo HEQ-piómetra}

La hiperplasia endometrial quística-piómetra tiene la edad como factor predisponente; los reportes indican que se da en pacientes hembras de entre 4 y 10 años. Asimismo, se ha reportado predisposición de ciertas razas como: Pastor Collie, Pastor Belga, Chow Chow, Bull Dog Francés, Pointer, Dogo Alemán, Rottweiler, Skye Terrier y San Bernardo, y con frecuencia se involucra a hembras caninas de razas grandes [6].

La HEQ-piómetra se produce principalmente por un exceso en el tiempo de exposición y por los niveles de progesterona en el endometrio; esto contribuye a disminuir la actividad miometrial, debido a una respuesta anormal del útero a las hormonas ováricas como progesterona y estrógenos [9], lo que facilita la retención de líquido [10]. La excesiva influencia progestacional o una respuesta exagerada a la progesterona ( $\mathrm{P} 4)$ inducen a que el tejido glandular uterino se vuelva quístico, edematoso y engrosado (HEQ); el drenaje queda impedido por la inhibición progestacional de la contractibilidad miometrial, que posteriormente brindará un ambiente adecuado y rico para el crecimiento bacteriano [11].

\section{Clasificación de la HEQ}

Según Boschera et al., los cambios patológicos úteroováricos de hembras caninas con hiperplasia endometrial se puede clasificar en cuatro tipos [12]:

Tipo I: se considera fisiológicamente normal, involucra un engrosamiento endometrial, así como numerosos quistes traslúcidos. Barton y Cain dicen que 
la HEQ tipo I no tiene complicaciones, ni signos clínicos de la enfermedad, y puede presentarse en cualquier periodo del ciclo estral, sobre todo en hembras caninas de edad media ( $<6$ años) [13].

Tipo II: se caracteriza por presentarse en la HEQ, con descarga vulvar mucoide; sólo ocurre durante el diestro, acompañado de leve leucocitosis. El endometrio se encuentra engrosado con elevaciones irregulares quísticas, y hay presencia de moco en el lumen uterino.

Tipo III: se considera patológica, dado que es quística y semejante a la tipo II, pues comienza en la etapa de diestro cuando el endometrio está bajo la acción de la $\mathrm{P} 4$. Ocurre entre los 20 y los 40 días después del estro, pero la HEQ y la actividad secretora de las glándulas endometriales producida por esta etapa del ciclo se mantienen funcionales, lo que provoca una acumulación de fluido uterino estéril y del lumen uterino, que se acompaña de una reacción inflamatoria aguda del endometrio.

Tipo IV: ocurre entre los 55 y los 90 días después del estro. Involucra endometritis quística crónica en la que el cérvix puede estar abierto o cerrado. Si el cérvix está abierto, se presenta descarga vaginal purulenta en la que los cuernos no se ven más grandes, pero sus paredes están engrosadas y dilatadas con apariencia de rosario; hay hipertrofia miometrial y fibrosis. Si el cérvix está cerrado, el útero se encuentra muy distendido, sus paredes delgadas dan la apariencia de útero grávido y está lleno de exudado purulento [12].

\section{Materiales y métodos}

Este estudio se llevó a cabo en Bucaramanga (Santander). Se obtuvieron úteros con diagnóstico de piómetra en dos clínicas asociadas a la Universidad Cooperativa: la Clínica de Pequeños Animales y la Clínica de Animales de Compañía. Se hicieron las respectivas mediciones con calibrador de peso total para medición de: los úteros sin contenido, el grosor de la pared del endometrio, el tipo de exudado, el tipo de olor, color y consistencia, la presencia de nódulos endometriales, los quistes endometriales y de la existencia o no de ovarios poliquísticos. No se tuvieron en cuenta variables asociadas a raza y edad dentro de los criterios de inclusión.

Todos estos datos fueron tabulados en Excel 2013 y luego fueron analizados en el programa Epi Info 7 para obtener un análisis univariable; también, se estableció la asociación de las variables objeto de estudio mediante una tabla de contingencia y una prueba de Fisher.

\section{Resultados}

Se hicieron las respectivas observaciones macroscópicas y las mediciones en 31 úteros con piómetra.

Tabla 1. Relación de hiperplasia endometrial quística (HEQ) y ovarios poliquísticos (OP)

\begin{tabular}{|c|c|c|c|}
\hline \multicolumn{3}{|c|}{ HEQ } & \\
\hline oP & No & Sí & Total \\
\hline No & 2 & 19 & $\mathbf{2 1}$ \\
\hline Row\% & $9,52 \%$ & $\mathbf{9 0 , 4 8 \%}$ & $100,00 \%$ \\
\hline Col\% & $50,00 \%$ & $70,37 \%$ & $67,74 \%$ \\
\hline Sí & 2 & 8 & $\mathbf{1 0}$ \\
\hline Row\% & $20,00 \%$ & $\mathbf{8 0 , 0 0 \%}$ & $100,00 \%$ \\
\hline Col\% & $50,00 \%$ & $29,63 \%$ & $32,26 \%$ \\
\hline Total & 4 & $\mathbf{2 7}$ & $\mathbf{3 1}$ \\
\hline Row\% & $12,90 \%$ & $87,10 \%$ & $100,00 \%$ \\
\hline Col\% & $100,00 \%$ & $100,00 \%$ & $100,00 \%$ \\
\hline
\end{tabular}

Fuente: elaboración propia

Del total de úteros con piómetra, el 90,48\% que tenían HEQ no tenían op, mientras que el $80 \%$ de los que sí presentaban op tenían HEQ.

Con la tabla de contingencia, se relacionó HEQ y op; y en la prueba de Fisher, el valor $p$ de la prueba fue de 0,577 , por lo cual no existe asociación significativa entre HEQ y OP $(p>0,05)$.

La frecuencia de presentación de HEQ fue del $87,10 \%$. Los úteros con contenido pesaban en promedio $272 \mathrm{~g}$ y sin contenido, $126 \mathrm{~g}$; el grosor de la pared endometrial medía en promedio $4 \mathrm{~mm}$ y se obtuvo un máximo de $10 \mathrm{~mm}$ en algunos úteros; el tipo de exudado más observado fue el sanguinopurulento (figuras 1 y 2 ) en $41,94 \%$ y purulento en $32,26 \%$; con color café cremoso en $41,94 \%$ y de consistencia cremosa en $35,48 \%$; la presencia de quistes endometriales representó el 83,9\% y se evidenció un hallazgo incidental compatible con masas uterinas en 9,68\% de los casos (figura 3). 


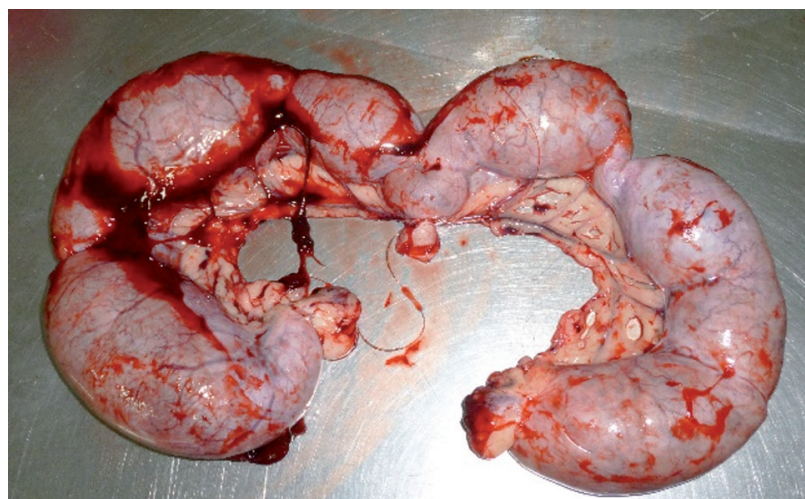

Figura 1. Dilatación de los cuernos uterinos, con áreas congestionadas y muy hemorrágicas

Fuente: elaboración propia

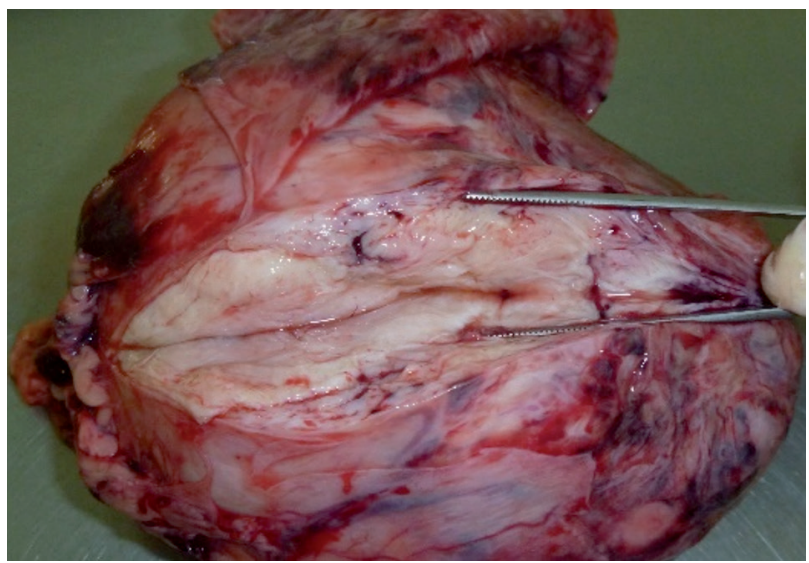

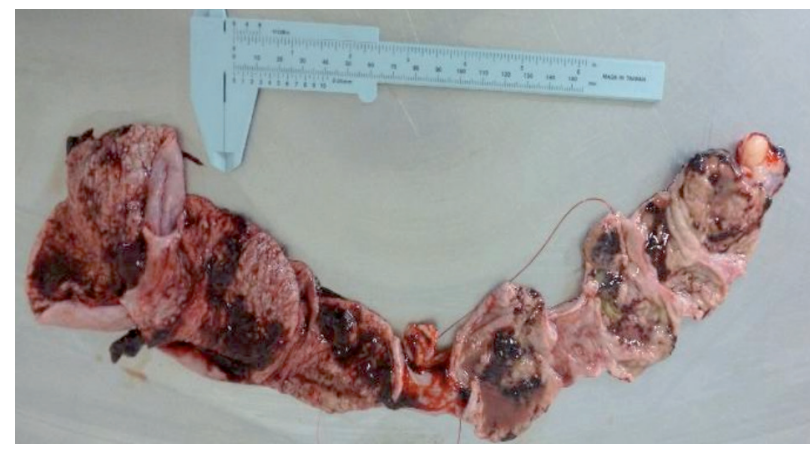

Figura 2. Mucosa uterina engrosada, con contenido sanguinolento

Fuente: elaboración propia

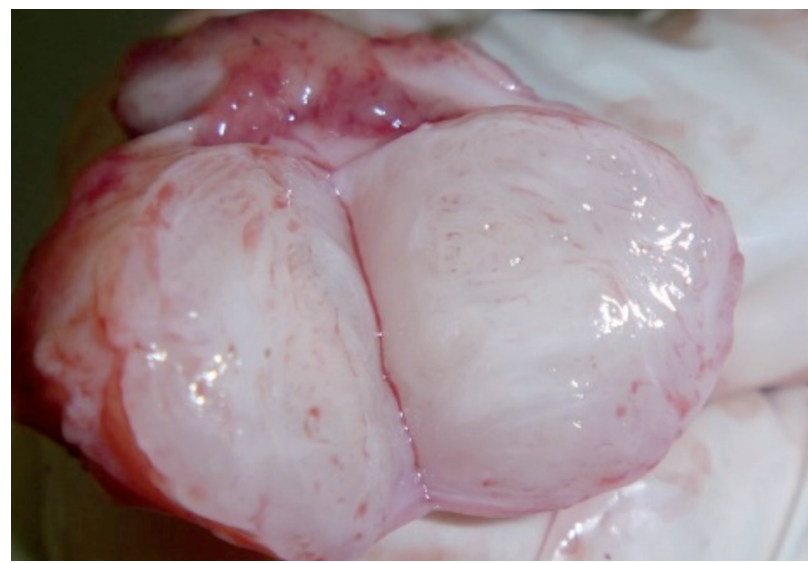

Figuras 3. Masas endometriales como hallazgo incidental, representadas en 9,68\%, sugieren posible neoplasia Fuente: elaboración propia

La frecuencia de presentación de los ovarios poliquísticos fue de 32,26\% (figuras 4 y 5). El tamaño de los ovarios varió de 10 a $14 \mathrm{~mm}$ de diámetro con un peso promedio de $3 \mathrm{~g}$; de los 31 casos observados, solo ocho casos tenían ovarios poliquísticos junto con hiperplasia endometrial quística.

\section{Discusión}

Se encontró que del total (31) de los casos estudiados con piómetra, la presentación del hallazgo de la hiperplasia endometrial quística (HEQ) es del $87,10 \%, y$ se determinó que el HEQ es el de mayor presentación para el presente estudio, resultados que coinciden con

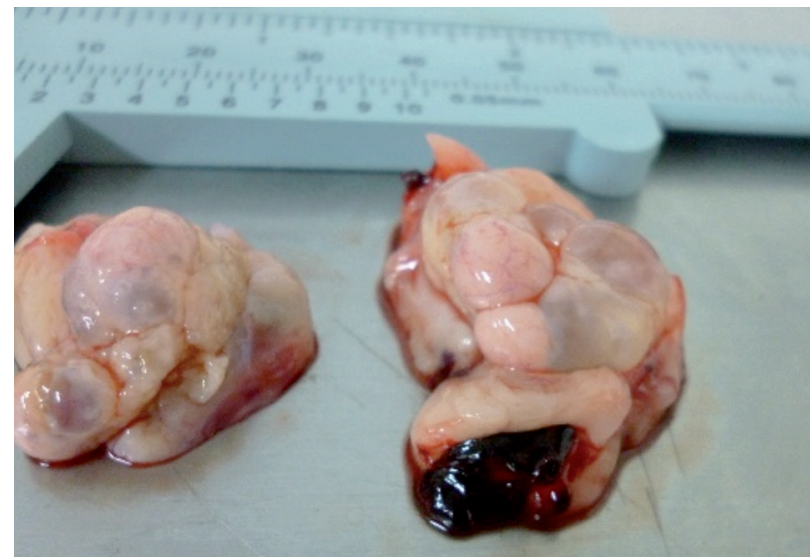

Figura 4. Múltiples quistes en ovarios con compromiso bilateral, de 2 a $4 \mathrm{~mm}$ de diámetro Fuente: elaboración propia 


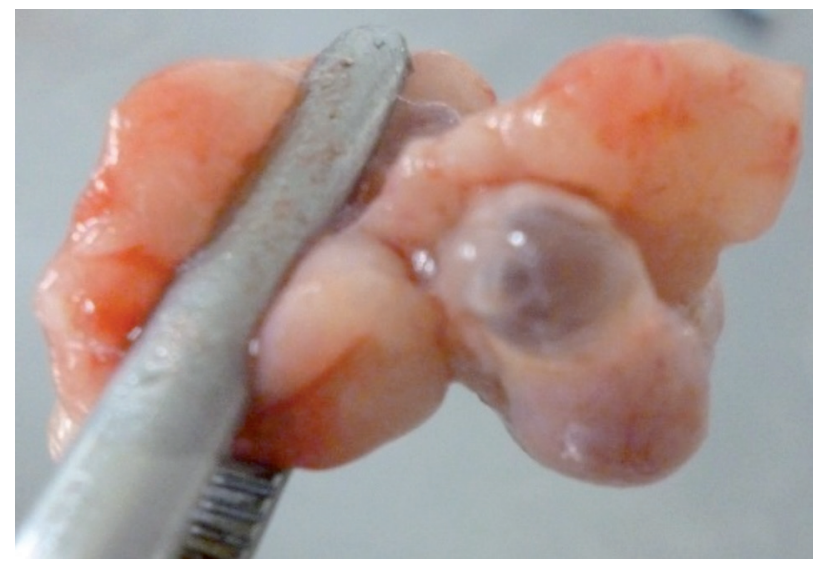

Figura 5. Ovario con quistes de 2 a $5 \mathrm{~mm}$ de diámetro o más grandes, múltiples quistes de $1 \mathrm{~mm}$ de diámetro Fuente: elaboración propia

lo reportado por Bigliardi et al. [10]. Sin embargo, Romagnoli y Concannon [14] determinaron que la HEQ es típicamente un hallazgo incidental y su incidencia natural no se conoce.

La presentación de quistes ováricos corresponde al $32,26 \%$ del total de los casos obtenidos y observados. La literatura no reporta la frecuencia de ovarios poliquísticos y tampoco la correlación con hiperplasia endometrial quística-piómetra. Solo coincide en que, entre las patologías concurrentes, pueden estar los ovarios quísticos, la HEQ y la piómetra por influencia hormonal común, como indican Báez y Ruiz [15]; o en que estas patologías se desarrollan por desequilibrios en los niveles hormonales de estrógenos y progesterona en fase de diestro [16], es decir, que el HEQ no requiere necesariamente la condición de la existencia de ovarios poliquísticos.

Reynoso [5], en su investigación, reportó presencia de quistes ováricos en el $68 \%$ de los casos y de HEQ en el 75\% de 16 casos analizados; sin embargo, no se encontró asociación entre los hallazgos, similar a lo reportado en el presente estudio.

La hiperplasia endometrial predispone la aparición de piómetra con exudado purulento [17], y como se evidenció aquí, está representada en el 32,26\%, con un contenido sanguinopurulento en un $41,94 \%$, de olor fétido y de consistencia viscosa. Estas características del exudado purulento se deben al contenido de bacterias que complican el estado de salud de la paciente.
La literatura indica que la infección del útero con agentes bacterianos causa morbilidad y mortalidad asociados con la piómetra [6].

Con respecto a úteros normales de diferentes individuos caninos, y con independencia de su edad, historial y etapa reproductiva, el grosor de la pared endometrial aparece aumentado debido a la hiperplasia que se genera en el endometrio, relacionada con la presencia macroscópica de quistes y nódulos endometriales, puesto que revela el tiempo de evolución de esta adaptación. Dichos cambios macroscópicos, según la clasificación de la hiperplasia, están asociados con la HEQ grados III y IV, en los que el endometrio se encuentra engrosado con elevaciones irregulares quísticas macroscópicas y con presencia de moco en el lumen uterino [13].

La existencia de masas compatibles con crecimiento como el leiomioma (figura 3 ) fue baja; aunque no fue una variable de inclusión para este estudio, la literatura sugiere que es la de mayor frecuencia [11], patología que, al ser benigna, solo se puede confirmar mediante observación histológica.

\section{Conclusiones}

Basados en los resultados de esta investigación, por medio de los hallazgos macroscópicos, la prueba de Fisher determinó que no existe asociación estadística entre la hiperplasia endometrial quística (HEQ) y los ovarios poliquísticos con el desarrollo de piómetra. Por su parte, relacionar el ciclo estral de las hembras caninas incluidas en este estudio o los antecedentes de la utilización de progestágenos no fue criterio de inclusión como variable objetivo, por lo cual no es preciso definir el estado reproductivo de los individuos y la posible causalidad relacionada con estos factores en el desarrollo de piómetra, lo que podrá ser incluido en estudios posteriores.

La piómetra tiene una presentación alta en hembras adultas enteras, pero identificar la fase del ciclo estral en la que ocurrre solo es posible mediante citología vaginal, junto con la medición de niveles hormonales séricos y el estudio histopatológico de las partes del útero y los ovarios, necesario para identificar los cambios en los tejidos. Estos métodos no fueron tenidos en cuenta para la propuesta inicial del presente estudio. 


\section{Referencias}

[1] Purswell BJ, Nikola PA. Reproducción en caninos y felinos domésticos. svv. 1997;9(2).

[2] Morales Z, Fidalgo LE. Patología médica veterinaria: libro de texto para la docencia de la asignatura. Galicia: Universidad de Santiago de Compostela; 2003.

[3] Verstegen J, Dhaliwal G, Verstegen-Onclin K. Mucometra Cystic Endometrial Hyperplasia, and Pyometra in the Bitch: Advances in Treatment and Assessment of Future Reproductive Success. Theriogenology. 2008;70(3):364-74.

[4] Albarracín JH, et al. Memorias Xi Encuentro Nacional e Internacional de las Ciencias Pecuarias ENICIP. Rev Colom Cienc Pecua. 2011;24(3).

[5] Reynoso R. Hiperestrogenismo en perros. Madrid: s. 1.; 2003.

[6] Silva RF, Loaiza AM. Piómetra en animales pequeños. Manizales: Universidad de Caldas; 2007.

[7] Nelson R, Couto G. Medicina interna de animales pequeños. Buenos Aires: Editorial InterMédica; 2000.

[8] Pérez-Marín CC., Molina L, Domínguez JM, Millán Y, Martín de las Mulas J. Adenomiosis uterina asociada a enfermedades ováricas y mamarias hormono dependientes en perra: un caso clínico. Recvet. Vol. II, No 05, Madrid, España, Mayo de 2007.
[9] Smith FO. Canine Pyometra. Theriogenology. 2006;66(3):610-2.

[10] Cunningham JG. Fisiología veterinaria. $2^{\mathrm{a}}$ ed. México D. F.: McGraw-Hill Interamericana; 1997.

[11] Bigliardi E, Parmigiani E, Caveman S, Luppi A, Bonati L, Corradi A. Ultrasonography and Cystic Hyperplasia-Pyometra Complex in the Bitch. Reprod Domest Anim. 2004;39(3):136-40.

[12] Boschera HD, Ducatelle R, Tshumala M. Is mechanically induced Cystic Endometrial Hyperplasia a suitable model for study of 555 spontaneously occurring CEH in the uterus of the bitch? Reproduction in Domestic Animal. 2002;37:152-7.

[13] Barton C, Cain J. Complejo hiperplasia endometrial quística-piómetra. En: Morgan R. Clínica de pequeños animales. $3^{\mathrm{a}} \mathrm{ed}$. Madrid: Harcourt Brace; 1999.

[14] Romagnoli S, Concannon PW. Uso clínico de progestinas en perras y gatas: una revisión. La Plata: Universidad Nacional de La Plata; 2005.

[15] Báez P, Ruiz I. Torsión uterina causada por un hemangioma cavernoso en una hembra canina. Rev Colom Cienc Pecua. 2006;19(3).

[16] Pyometra, Cystic Endometrial Hiperplasia-Pyometra Complex [internet]. s. f. Disponible en: http:// www.greenvillevet.com/GVC_Pyometra.pdf

[17] Pretzer SD. Clinical Presentation of Canine Pyometra and Mucometra: A review. Theriogenology. 2008;70(3):359-63. 